\title{
Notes on the result of solutions of the equilibrium equations
}

\author{
Gaiping $\mathrm{He}^{1}$, Lihong Wang ${ }^{2}$ and Ben Rodrigo ${ }^{3^{*}}$
}

"Correspondence: ben.rodrigo@aol.com

${ }^{3}$ Instituto de Matemática y Física, Universidad de Talca, Talca, Chile Full list of author information is available at the end of the article

\section{Abstract}

In this short note, we correct some expressions obtained by War y et al. (Bpund. Value Probl. 2015:230, 2015). The corrected expressions will be use ul fo valuating the boundary behaviors of solutions of modified equilibrium eq ions vrrn finite mass subject. Moreover, the correction of Theorem 2.1 is als given.

Keywords: Boundary behavior; Equilibrium equation; te mass

\section{Introduction}

The origin of our work lies in Wang et at $\left[y_{1}\right.$. 11], they investigated slow equilibrium equations with finite mass subject to a homugeneous Neumann-type boundary condition. As an application, the exister ce on 'utions for Laplace equations with a Neumann-type boundary condition was aiso ovea which has recently been used to study the Cauchy problem of Laplace ecatation by $\mathrm{ng}$ [2].

However, there exis an $n$ sprints and erroneous expressions in [1]. Firstly, we correct some misr nts in S. 2. Then we correct erroneous expressions in Sect. 3. The corrected ver ion $; 1$ be useful for evaluating the boundary behaviors of solutions of the equilibri am equation, with finite mass subject. Finally, we correct Theorem 2.1 in Sect. 4. The pr ent notation and terminology is the same as in [1].

\section{2 me misprints}

Wt re ndebted to the anonymous reviewer for pointing out to us that the following shor $1 \mathrm{~d}$ also be corrected in [1].

(1) A correct version of Abstract reads as follows.

The aim of this paper is to study the models of rotating stars with prescribed angular velocity. We prove that it can be formulated as a variational problem. As an application, we are also concerned with the existence of equilibrium solution.

(II) $\mathbb{R}^{4}$ and $x_{4}$ should be written as $\mathbb{R}^{3}$ and $x_{3}$, respectively.

(III) Introduction: instead of " $3-\mathrm{D}$ ", there should be "4-D".

(IV) Some main references [1, 2, 3] should be corrected as follows:

[1] Auchmuty, G, Beals, R: Variational solutions of some nonlinear free boundary problems. Arch. Ration. Mech. Anal. 43, 255-271 (1971)

[2] Li, Y: On Uniformly Rotating Stars. Arch. Ration. Mech. Anal. 115, 367-393 (1991)

[3] Deng, Y, Yang, T: Multiplicity of stationary solutions to the Euler-Poisson equations. J. Differ. Equ. 231, 252-289 (2006)

\section{照 Springer}


(V) On page 2, line 12: instead of " $P$ :", there should be " $P_{1}:$ ".

\section{Corrected expressions}

We find that [1, inequality (2.4)] is not correct and should be modified as (the sign before the function " $\left(\frac{M_{1}}{M}\right)^{5 / 3}$ " should be “"

$$
\begin{aligned}
h_{M}-F(\rho) \leq & \left(1+\left(\frac{M_{1}}{M}\right)^{5 / 3}-\left(\frac{M_{2}}{M}\right)^{5 / 3}-\left(\frac{M_{3}}{M}\right)^{5 / 3}\right) h_{M}+\frac{C_{1}}{R_{2}} \\
& +\frac{C_{3}}{R_{2}}\left\|\nabla \Phi_{2}\right\|_{2} \\
\leq & C_{4} h_{M} M_{1} M_{3}+\frac{C_{5}}{R_{2}}\left(1+\left\|\nabla \Phi_{2}\right\|_{2}\right) .
\end{aligned}
$$

Therefore, the expressions in [1] that are derived by using [1, ine ${ }_{1} \mathrm{u}_{a}$ ( $\left.(2.4)\right]$ need to be corrected. Specifically, [1, inequality (2.8)] should be modified $n \mathrm{~s}$

$$
\begin{aligned}
-C_{4} h_{M} \delta_{0} M_{n, 3} \leq & C_{4} h_{M} M_{n, 1} M_{n, 3} \\
\leq & \left.\frac{C_{5}}{R_{2}}\left(1+\left\|\nabla \Phi_{0,2}\right\|_{2}\right)+C_{5} \| \nabla \Phi_{n, 2}-\nabla \Phi_{0,2, \| 2}\right) \\
& +\left|F\left(T \rho_{n}\right)-h_{M}\right|+\rho_{0} .
\end{aligned}
$$

These corrections will be useful for the reac who want to use [1, Theorem 2.1] to evaluate the boundary behavior of so. is of $_{\text {of }}$ the equilibrium equations with finite mass subject.

\section{Corrected Theorem 2.1}

A correction of Theor $\mathrm{n} 2.1$ in [1] reads as follows.

Theorem 2.1 $\mathrm{L}$ hold. Let $\left(\rho_{n}\right)_{n=1}^{\infty} \in \mathcal{A}_{M}$ be a minimizing sequence of $F$. Then there exists a suhsequercr, stul denoted by $\left(\rho_{n}\right)_{n=1}^{\infty}$, and a sequence of translations $T \rho_{n}:=\rho_{n}(\cdot+$ $\left.a_{n} e_{3}\right)$, here $i_{n}$ are onstants, and $e_{3}=(0,0,1)$, such that

$$
F\left(\rho_{0}\right)=\operatorname{Aff}_{\mathcal{A}_{M}} F(\rho)=h_{M}+\rho_{0}
$$

ai) $T \rho_{n} \rightarrow \rho_{0}$ weakly in $L^{\frac{4}{3}}\left(\mathbb{R}^{3}\right)$. For the induced potentials, we have $\nabla \Phi_{T \rho_{n}} \rightarrow \nabla \Phi_{\rho_{0}}$ veakly in $L^{2}\left(\mathbb{R}^{3}\right)$.

Proof Define

$$
I_{l m}:=\iint \frac{\rho_{l}(x) \rho_{m}(y)}{x-y} d y d x
$$

for $l, m=1,2,3$.

Let $\rho=\rho_{1}+\rho_{2}+\rho_{3}$, where $\rho_{1}=\chi_{B_{R_{1}}} \rho, \rho_{2}=\chi_{B_{R_{1}, R_{2}}} \rho$, and $\rho_{3}=\chi_{B_{R_{2}}} \rho$. So we have

$$
F(\rho)=F\left(\rho_{1}\right)+F\left(\rho_{2}\right)+F\left(\rho_{3}\right)-I_{12}-I_{13}-I_{23} .
$$


Choosing $R_{2}>2 R_{1}$, we have

$$
I_{13} \leq 2 \int_{B_{R_{1}}} \frac{\rho(x)}{R_{1}} d x \int_{B_{R_{2}, \infty}} \frac{\rho(y)}{|y|^{2}} d y \leq \frac{C_{1}}{R_{2}} .
$$

Next, we estimate $I_{12}$ and $I_{23}$ :

$$
\begin{aligned}
I_{12}+I_{23} & =-\int \rho_{1} \Phi_{2} d x-\int \rho_{2} \Phi_{3} d x=\frac{1}{4 \pi g} \int \nabla\left(\Phi_{1}+\Phi_{3}\right) \cdot \nabla \Phi_{2} d x \\
& \leq C_{2}\left\|\rho_{1}+\rho_{3}\right\|_{\frac{6}{5}}\left\|\nabla \Phi_{2}\right\|_{2} \leq C_{3}\left\|\nabla \Phi_{2}\right\|_{2} .
\end{aligned}
$$

If we define $M_{l}=\int \rho_{l} d x$, then it is easy to see that $M=M_{1}+M_{2}+M_{3}$.

The remaining proofs are carried out in the same way as for Theorem -1 in $\quad$ J, except that instead of the erroneous expressions (2.4) and (2.8), we have to their co, rected versions given in Sect. 2.

\section{Conclusions}

In this note, we corrected some expressions obtained Wang t. 11]. The corrected expressions will be useful for evaluating the boundary b ha. of solutions of the equilibrium equations with finite mass subject. Moreover, the correction of Theorem 2.1 was also given.

\section{Acknowledgements}

The authors would like to thank Professor D. Simms orrrigin to our attention that the expressions in [1] are not true in general. The authors are also grateful to the anonymo vie ver for his valuable observation.

\section{Funding}

This work was supported by FONDE.t.T (No. 11, /9).

Competing interests

The authors declare that they no competing interests.

\section{Authors' contributio}

BR drafted the manuscript. 9 LW helped to revise the written English and revised the manuscript according to the referee repor+ ' autho, readand approved the final manuscript.

\section{Authorn ils}

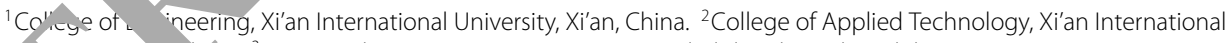
ersity, Xi'ar ina. ${ }^{3}$ Instituto de Matemática y Física, Universidad de Talca, Talca, Chile.

\section{Publis,rer's Note}

Splinger Nature remains neutral with regard to jurisdictional claims in published maps and institutional affiliations.

Received: 28 September 2017 Accepted: 5 March 2018 Published online: 17 April 2018

\section{References}

1. Wang, J., Pu, J., Huang, B., Shi, G.: Boundary value behaviors for solutions of the equilibrium equations with angular velocity. Bound. Value Probl. 2015, 230 (2015)

2. Wang, Y:: A regularization method for the Cauchy problem of Laplace equation. Acta Anal. Funct. Appl. 19(2), 199-205 (2017) 